\title{
Les coopérations au développement durable en question dans les pays du Sud
}

Cooperation in Sustainable Development at Question in Countries of the South

\section{Géraldine Froger}

\section{OpenEdition}

12 Journals

\section{Édition électronique}

URL : http://journals.openedition.org/developpementdurable/8364

DOI : 10.4000/developpementdurable.8364

ISSN : 1772-9971

Éditeur

Association DD\&T

\section{Référence électronique}

Géraldine Froger, «Les coopérations au développement durable en question dans les pays du Sud", Développement durable et territoires [En ligne], Vol. 1, n 1 | Mai 2010, mis en ligne le 07 mai 2010, consulté le 19 avril 2019. URL : http://journals.openedition.org/developpementdurable/8364 ; DOI : 10.4000/developpementdurable.8364

Ce document a été généré automatiquement le 19 avril 2019.

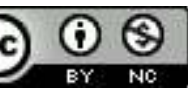

Développement Durable et Territoires est mis à disposition selon les termes de la licence Creative Commons Attribution - Pas d'Utilisation Commerciale 4.0 International. 


\title{
Les coopérations au développement durable en question dans les pays du Sud
}

\author{
Cooperation in Sustainable Development at Question in Countries of the South
}

\author{
Géraldine Froger
}

1 La coopération décentralisée qui renvoie à l'action extérieure des collectivités territoriales est venue s'inscrire de manière de plus en plus affirmée dans le champ de l'appui au développement des pays du Sud (Marie, 2005), aux côtés de la coopération « classique » déjà mise en oeuvre par les États dans le cadre de la coopération bilatérale (s'exerçant de pays à pays) ou par les organismes multilatéraux, i.e. regroupant plusieurs pays: Union européenne, organismes des Nations unies, institutions financières internationales (Banque Mondiale, Fonds Monétaire International, etc.), dans le cadre de la coopération multilatérale. Quelles réalités recouvre la coopération décentralisée ? Quels en sont les champs d'action? Quels en sont les avantages par rapport aux formes classiques de coopération? Quelles en sont les ambiguïtés? L'objectif de cet article est d'abord de resituer la coopération décentralisée par rapport aux autres formes classiques de coopérations (1). Nous mettrons ensuite l'accent sur la portée et les ambiguïtés de la coopération décentralisée comme appui au développement durable dans les pays du Sud (2). Cela nous amènera, enfin (3), à nous intéresser plus particulièrement aux politiques de développement durable soutenues par les formes classiques de coopération, en analysant la portée et les limites de la gouvernance décentralisée des ressources naturelles, à l'aide de l'exemple de Madagascar comme cas d'étude.

\section{Coopération décentralisée : définition et état des lieux}

2 Intuitivement, l'expression de coopération décentralisée renvoie à l'action extérieure des collectivités locales. Cette acception large englobe des actions ne s'exerçant pas dans le 
cadre de conventions (opérations de promotion économique et culturelle par exemple), et ne permet pas une distinction très claire avec la mission de l'État en matière internationale. La loi d'orientation du 6 février 1992 d'administration territoriale de la République a consacré une acception désormais reconnue et plus restrictive: dans ce cadre, la coopération décentralisée comprend l'ensemble des actions de coopération internationale réalisées par les collectivités territoriales françaises et leurs groupements, sur la base de conventions, avec des collectivités publiques étrangères ${ }^{1}$ (Tulard, 2006). En faisant le point sur les initiatives prises par les collectivités territoriales françaises dans le domaine de la coopération décentralisée, on constate que les actions extérieures ${ }^{2}$ des régions, des départements, des villes et de leurs groupements sont très diverses dans leur forme et dans leurs objets. Leurs actions sont souvent appuyées par des acteurs multiples: organisations non gouvernementales (ONG), associations, bailleurs de fonds internationaux, experts ${ }^{3} \ldots$... Que ce soit dans une acception large ou plus précise, la coopération décentralisée regroupe des pratiques très diverses que l'on peut regrouper de la manière suivante :

D'abord, les relations basées sur des liens à vocation amicale, culturelle, linguistique ou économique, dans la continuité des jumelages des années $1950^{4}$. Ensuite, les coopérations transfrontalières qui visent à mettre en œuvre des infrastructures ou des services communs dans le contexte européen sur la base d'accords internationaux et d'instruments juridiques adéquats (Tulard, 2006). En reprenant les travaux de Bonnin (2008), le premier texte international relatif à cette coopération transfrontalière est la convention-cadre européenne sur la coopération transfrontalière entre les collectivités territoriales. Cette convention, ouverte à la signature des États membres du Conseil de l'Europe, à Madrid, le 21 mai 1980, a pour but d'encourager et de faciliter la conclusion d'accords entre régions et communes, de part et d'autre d'une frontière, dans les limites de leurs compétences. Par cette convention, les États se sont engagés à "faciliter et à promouvoir la coopération transfrontalière». Les accords prévus par cette convention peuvent s'étendre entre autres au développement régional, à la protection de l'environnement, ou encore à l'aménagement des infrastructures et des services publics. Cependant, élaborée à la fin des années 1970, cette convention correspond à une époque où la coopération transfrontalière apparaît encore comme une menace à la souveraineté des États. Partant, elle n'implique aucune obligation précise pour les États. De plus, elle ne reconnaît pas officiellement un droit des collectivités à conclure des accords de coopération transfrontalière. Le protocole additionnel de 1995 signé ultérieurement vise à renforcer la convention-cadre en reconnaissant expressément, sous certaines conditions, le droit des collectivités territoriales à conclure des accords de coopération transfrontalière. Il a permis le développement de projets novateurs en matière de développement durable. À noter que les collectivités territoriales sont de plus en plus impliquées dans cette forme de coopération, dans l'Union européenne et son voisinage et sont appelées à développer leur présence dans les programmes communautaires et multilatéraux. Enfin, les différentes formes d'aide au développement qui, même si elles sont plus faibles en nombre, n'en restent pas moins importantes. Marie (2005) note une évolution de la coopération décentralisée qui passe progressivement de l'assistance humanitaire au développement à proprement parler. Le développement durable est une des thématiques qui monte en puissance dans le cadre de cet appui au développement des pays du Sud (IEPF, 2007). 
4 Avant de se focaliser sur les champs d'intervention privilégiés de la coopération décentralisée en appui au développement des pays du Sud (en complément des champs d'intervention des formes "classiques » de coopération), il est intéressant de montrer quelle en sont les zones d'intervention principales ainsi que les efforts financiers consacrés.

5 Les zones d'intervention privilégiées par les collectivités, hors pays d'Europe où leur présence est conséquente, sont l'Afrique francophone, puis la Chine et l'Asie du Sud-Est. Elles ont une présence encore limitée mais en progression en Amérique du Sud et ont fait quelques percées en Afrique lusophone et anglophone, enfin une forte présence dans les pays d'Europe médiane (Pologne, Roumanie, Hongrie...) $)^{5}$.

6 D'après la base de données de la Commission nationale de la coopération décentralisée $(\mathrm{CNCD})^{6}$, il existe quelque 3800 collectivités territoriales françaises (régions, départements, communes et structures intercommunales) qui mènent des projets de coopération à l'international totalisant près de 8000 coopérations localisées dans 132 pays. Les pays de l'Union européenne arrivent largement en tête avec 4600 partenariats. Les pays situés hors de l'Union européenne accueillent environ 3400 projets de coopération, répertoriés dans le futur Atlas de la coopération décentralisée. Dans cet ensemble, sont distingués : premièrement, la zone dite de «solidarité prioritaire » (ZSP) comprenant 54 pays avec lesquels la France entretient des relations privilégiées, où sont dénombrés 1115 liens, soit 32,8\% du total hors Union européenne. Ils se répartissent de la manière suivante: Afrique subsaharienne et océan indien, avec une concentration sur le Burkina Faso, le Mali et le Sénégal; Afrique du Nord/Maghreb ; Asie du Sud-Est, principalement au Vietnam; Caraïbes. Deuxièmement, les pays développés non européens où sont dénombrés 870 liens, soit $25,6 \%$ du total hors Union européenne. Troisièmement, pour l'Europe médiane et orientale, ont été comptés 1067 liens, soit $31,4 \%$ du total hors Union européenne (avec une concentration sur la Roumanie, la Pologne, la République tchèque, un développement récent sur la Hongrie et une présence significative en Russie). Quatrièmement, pour les pays émergents, intermédiaires ou en développement hors ZSP, ont été comptabilisés 380 liens, soit $11,2 \%$ du total hors Union européenne.

7 S'ajoutent à ces partenariats, recensés dans le futur Atlas de la CNCD, de nombreuses opérations unilatérales des régions, des départements, des communes et de structures intercommunales, en matière de promotion économique ou culturelle à l'étranger, ainsi que des relations de partenariat en cours pouvant déboucher ultérieurement sur la conclusion de conventions de coopération décentralisée.

Récemment, une tendance se développe pour une cohérence plus affirmée des actions réalisées en fonction de logiques thématiques (gouvernance de proximité, aménagement du territoire, services publics locaux, eau, développement durable) dans la continuité des engagements de Johannesburg et de Kyoto, avec un développement des réflexions à l'échelle des régions en liaison avec l'ensemble des acteurs du territoire.

9 En termes financiers, les sommes consacrées par les collectivités territoriales à l'action extérieure ont été estimées à 230 millions d'euros. La moitié environ, soit 115 millions d'euros, a été destinée à des partenaires localisés dans les pays en développement (CNCD, 2009). Les cofinancements accordés par le Ministère des Affaires étrangères et européennes ont été de 11,77 millions d'euros (soit 10\% environ des dépenses liées aux actions de développement des collectivités territoriales) en 2002, de 12,5 millions d'euros 
dans le cadre des appels à projet triennaux 2007-2009, et de 4,22 millions d'euros dans le cadre des appels à projet 2008. La répartition des dépenses entre niveaux de collectivités et par destination se présente comme suit (tableau 1).

\begin{tabular}{|c|c|c|}
\hline \multicolumn{2}{|c|}{$\begin{array}{l}\text { Dépenses totales en matière d'actions extérieures } \\
\text { en } 2002\end{array}$} & \multirow{2}{*}{$\begin{array}{l}\text { Dont actions pour le développement } \\
\begin{array}{l}33 \text { M euros (environ } 40 \% \text { de leurs } \\
\text { dépenses) }\end{array}\end{array}$} \\
\hline Régions & $82 \mathrm{M}$ euros & \\
\hline Part total & $36 \%$ & \\
\hline Départements & $24 \mathrm{M}$ euros & $\begin{array}{l}13 \mathrm{M} \text { euros (environ } 55 \% \text { de leurs } \\
\text { dépenses) }\end{array}$ \\
\hline Part total & $10,6 \%$ & \\
\hline Communes et groupements & $122 \mathrm{M}$ euros & $\begin{array}{l}69 \mathrm{M} \text { euros (environ } 56 \% \text { de leurs } \\
\text { dépenses) }\end{array}$ \\
\hline Part du total & $53,4 \%$ & \\
\hline Total en euros & $230 \mathrm{M}$ euros & $\begin{array}{l}115 \mathrm{M} \text { euros (environ } 50,3 \% \text { de leurs } \\
\text { dépenses) }\end{array}$ \\
\hline Total en \% & $100 \%$ & \\
\hline
\end{tabular}

\section{Portée et ambiguïtés de la coopération décentralisée dans le champ du développement durable}

12 Malgré la diversité des formes de coopérations décentralisées (jumelages; expertise, appui technique, assistance à maîtrise d'ouvrage ; formations ; fourniture de matériels ou d'ouvrages; subventions; coopération transfrontalière ou soutien au collectivités du Sud), celles-ci se fédèrent principalement autour du développement local, ce dernier comprenant (1) le développement économique animant les partenariats Nord/Sud ; (2) le développement urbain et rural; (3) et le développement durable qui intègre les problématiques environnementales. En mettant plus particulièrement l'accent sur les champs principaux des partenariats Nord/Sud en matière de coopération décentralisée, ces derniers concernent : (1) l'appui institutionnel et l'appui à la décentralisation ; (2) le développement urbain ; (3) le développement rural ${ }^{7}$; (4) l'action sanitaire et sociale ; (5) la culture, l'éducation et la recherche (Tulard, 2006). Notons également qu'un champ d'action non négligeable de la coopération décentralisée renvoie au tourisme responsable et solidaire (Joly et Gallet, 2006 ; DGCID, 2006). Houzir (2007:16) note que si le contenu des coopérations décentralisées est très divers, il concerne: soit un renforcement de 
capacités de gestion des services publics locaux dans l'ensemble des domaines de compétence des collectivités territoriales (appui institutionnel, assistance à maitrise d'ouvrage, aide à la mise en place de services de base, formations, aménagement du territoire, gestion à la culture et au patrimoine, ingénierie de projets, intercommunalité). Soit, notamment depuis les cinq dernières années, des projets privilégiant des thématiques plus globales ayant trait au développement durable (développement territorial, tourisme responsable et solidaire, co-développement...) ${ }^{8}$.

Ainsi, l'évolution contemporaine des interventions de la coopération décentralisée devrait illustrer le passage d'une logique de développement conçu sous la forme de projets plus ou moins sectoriels (puits, écoles, dispensaires,...) correspondant à des besoins ponctuels des collectivités du Sud vers une véritable logique de développement durable local, conçu sous la forme de programmes multi-sectoriels.

Engageant les collectivités locales et territoriales des pays du Nord dans des actions de développement en partenariat avec des collectivités des pays du Sud, la coopération décentralisée a d'emblée été présentée comme complémentaire aux formes classiques de coopération car comme le souligne Marie (2005): «les partenaires sont bien identifiés; ils sont censés être plus proches les uns des autres et des réalités locales que les méga-institutions lointaines et impersonnelles que sont les États et les institutions internationales; ils peuvent entretenir, en principe, des relations plus directes, plus souples, plus humaines, et plus sensibles aux spécificités des populations; ils peuvent, théoriquement, promouvoir des modalités du développement mieux adaptées aux conditions particulières de lieux et d'environnement social et culturel; ils sont censés échapper aux lourdeurs bureaucratiques, financières et juridiques des coopérations classiques et, dans un contexte nouveau de démocratisation et de décentralisation au Sud (à partir des années 1990), les collectivités françaises peuvent apporter leurs compétences spécifiques en matière d'appui à la gestion municipale et à la promotion d'un développement décentralisé » (ibid. : 22).

Par ailleurs, la coopération décentralisée a bénéficié d'une légitimité supplémentaire suite à la remise en cause des États des pays en développement critiqués pour leur inefficacité, les corruptions constatées, le développement de projets " pharaoniques »... et à celle des organisations internationales contestées dans leurs démarches «technocratiques» (Froger et Andriamahefazafy, 2003). Dans ce contexte, elle peut apparaître comme étant capable de promouvoir une conception plus modeste et plus réaliste du développement, mieux adapté aux situations concrètes et aux conditions locales, mieux approprié aux besoins et aux moyens des populations, donc mieux « appropriable » par les bénéficiaires dans une certaine indépendance vis-à-vis des États et des classes dirigeantes dénoncées pour leurs stratégies de rente. Plusieurs articles de la revue Liaison Energie-Francophonie (IEPF, 2007) illustrent d'ailleurs les avantages de la coopération décentralisée dans le champ du développement durable pour la plupart des raisons mentionnées ci-dessus.

16 La coopération décentralisée dans les champs du développement en général et du développement durale en particulier n'en reste pas moins confrontée à plusieurs ambiguïtés.

17 Selon Marie (2005:35), dans la mesure où la coopération décentralisée s'organise et se coordonne sous l'impulsion de différents acteurs (nationaux et internationaux), elle se retrouve dans une « logique de bureaucratisation (avec une multiplication des instances hiérarchisées)» et de relative dépendance vis-à-vis de l'État (ministères ou agences) ou 
des organismes multilatéraux (Union européenne, bailleurs de fonds internationaux). Si elle dispose de plus de moyens, ses capacités de conception et d'action autonomes ainsi que la souplesse souhaitée de son fonctionnement s'en trouvent réduites.

La coopération décentralisée fait aussi souvent appel à des sources financières extérieures de financement. Cet appel à l'intervention de différents partenaires financiers multiplie et alourdit les procédures et règles en matière de contrôle budgétaire et de gestion comptable des montages financiers; de plus, il faut pouvoir faire état de résultats et justifier les actions, ainsi que les dépenses auprès des donateurs. Ces éléments viennent renforcer une logique de réalisations rapidement visibles de « projets bancables » inscrits dans une logique de court terme au détriment d'une logique de programmation de plus long terme. «La complexité des montages institutionnels et l'obligation de visibilité des résultats sont deux logiques qui peuvent se concilier (certes au prix d'une certaine lourdeur bureaucratique et du renchérissement des dépenses de fonctionnement au détriment des investissements...) mais qui sont assurément contradictoires avec les principales missions spécifiques attendues de la coopération décentralisée: (......) appui à la gestion décentralisée des affaires publiques, renforcement des capacités locales du Sud, programmation d'un développement [durable] adapté aux conditions locales, toutes missions qui requièrent des actions de longue haleine, aux résultats peu tangibles à court terme et difficilement chiffrables en termes comptables " (ibid. : 40).

Ainsi, malgré l'évolution souhaitée de la coopération décentralisée vers des démarches plus globales, les actions réalisées dans la majorité des partenariats n'en restent pas moins marquées par une approche sectorielle (Houzir, $2007: 17)^{9}$. Celle-ci se traduit : (1) par une concentration des projets dans un petit nombre de secteurs (échanges socioculturels, action sociale et jeunesse, santé, éducation et formation); (2) par un faible investissement dans plusieurs secteurs d'intervention (création ou développement d'activités économiques, hormis en faveur des femmes, l'aménagement urbain ou rural, la gestion des services d'intérêt collectif [eau, assainissement,...] alors que ces derniers sont mentionnés comme prioritaires par plusieurs acteurs [responsables des collectivités du Sud, organisations d'habitants, associations locales...]). À titre illustratif, à Madagascar il existe actuellement 18 partenariats entre régions du Nord et régions malgaches, 17 partenariats entre départements du Nord et départements malgaches, 3 partenariats entre groupements et 27 partenariats entre communes (source : site internet de la CNCD). Les actions portent essentiellement sur l'éducation, la formation, les projets hydrauliques, l'aide humanitaire, le jumelage... Les approches plurisectorielles insérées dans une logique plus globale de développement local durable restent incontestablement minoritaires à l'heure actuelle dans l'ensemble des pays du Sud.

À l'issue de ce bilan, force est de constater que l'élaboration de politiques de développement durable et/ou l'élaboration de programmes sur un horizon de plus long terme relèverait actuellement pour l'essentiel des formes « classiques » de coopération. Il s'agit alors d'évaluer la portée et les limites des nouvelles formes d'intervention des politiques classiques de coopération, complémentaires à celles de la coopération décentralisée, dans les champs de l'appui à la décentralisation et de l'appui au développement durable, notamment au travers de la "gouvernance décentralisée des ressources naturelles ». 


\section{La gouvernance décentralisée des ressources naturelles en question}

21 Même si, avant le début des années 1990, les bailleurs de fonds multilatéraux et bilatéraux s'intéressent aux questions environnementales et de développement durable, c'est essentiellement à partir de cette période que leurs interventions dans ces domaines se renforcent dans les pays du Sud (Froger et Andriamahafazafy, 2003). De même, c'est au cours des années 1990 que la nouvelle philosophie des programmes d'aide influençant les politiques et les projets environnementaux mis en oeuvre dans les pays en développement s'appuie sur le recours à la société civile et au développement participatif pour éviter la déresponsabilisation des pays récepteurs de l'aide en développement et donc favoriser l'appropriation des politiques par les gouvernants et les populations ${ }^{10}$. On rejoint l'une des préoccupations de la coopération décentralisée (thème de l'appropriation et de «l'appropriabilité ») mentionnée plus haut.

22 Pour illustrer la portée et les ambiguïtés de la décentralisation appliquée aux ressources naturelles (qualifiée de gouvernance décentralisée des ressources naturelles), nous prendrons l'exemple de Madagascar avant de revenir sur les enseignements que l'on peut en tirer pour les pays en développement en général.

23 Sans revenir sur les différents temps de la politique environnementale malgache ${ }^{11}$, c'est au début des années 1990 que se popularise l'idée d'un transfert de gestion des ressources naturelles aux communautés locales et d'une gouvernance décentralisée soutenus par des formes classiques de coopération (dans des zones d'intervention spécifiques) ${ }^{12}$, pour pallier les défaillances de l'État et de ses services déconcentrés en matière de protection de l'environnement et de conservation de la biodiversité, ainsi que pour répondre aux problèmes de feux de brousse (à Madagascar, par exemple).

24 À Madagascar, les fondements de cette gouvernance ont été posés avec l'adoption en 1996 de la loi sur le transfert de la gestion des ressources naturelles d'un terroir aux communautés de base. Cette loi $96-025$ relative à la gestion locale sécurisée (GELOSE) ${ }^{13}$ repose sur trois grands principes (Belvaux et Rabearisoa, 2006) :

25 - l'établissement d'un contrat entre différentes parties prenantes : État via les services techniques déconcentrés de l'administration, commune et communautés locales de base bénéficiaires ;

26 - l'intervention d'un médiateur, personne extérieure, ayant pour principaux objectifs de déceler les conflits potentiels pouvant remettre en cause le contrat établi et de faciliter la négociation d'objectifs communs (dans une démarche patrimoniale) qui seront transformés en règles de gestion respectueuses de l'environnement et des intérêts des populations ;

27 - la possibilité pour une communauté d'obtenir une sécurisation foncière relative - « constatation publique, collective et contradictoire des occupations foncières sur l'ensemble $d u$ territoire concerné : ce n'est ni une immatriculation, ni un cadastrage. Les droits sont convenus entre les parties mais ne sont pas garantis par l'État de façon absolue et inattaquable » (ibid. : 3).

28 Ce dispositif de gouvernance décentralisée rencontre plusieurs difficultés. La mise en œuvre de la gestion décentralisée sur le terrain malgache s'est heurtée au cours des premières années à des obstacles qui, pour la plupart d'entre eux, n'ont pas été encore aujourd'hui levés. Parmi ces derniers, on peut citer (Belvaux et Rabearisoa, 2006) : 
29 - une élaboration lente et sans respect chronologique des textes d'application de la loi GELOSE dont certains n'ont pu encore être adoptés - ce qui empêche un réel développement des contrats de transfert de gestion;

30 - la parution, en février 2001, du décret sur la Gestion contractualisée des forêts (décret 2001-122, spécifique au transfert des ressources forestières relevant du domaine privé de l'État) portant préjudice à l'application de la loi GELOSE, dans la mesure où le contrat n'est signé qu'entre deux parties (État et communautés bénéficiaires), qu'il n'impose pas l'intervention d'un médiateur et qu'il ignore les procédures en matière de sécurisation foncière relative ;

31 - des dispositifs d'intervention des opérateurs et des organismes d'appui insuffisamment harmonisés sur le terrain : si le cadre juridique balise ces interventions, «le premier guide à destination des techniciens ne parait qu'en 1999, et il faut attendre 2002-2003 pour que soient élaborés des outils à destination des opérateurs et des organismes d'appui. À ce titre, la parution de 'directives générales' (toujours absentes à ce jour) indiquant les procédures à suivre tout au long $d u$ processus, avec une déclinaison par ressources spécifiques, n'a jamais été engagée alors qu'elle aurait dû constituer une priorité » (ibid. : 4);

32 - la procédure de sécurisation foncière relative estimée par les opérateurs et organismes d'appui comme une contrainte: considérée comme trop complexe (difficulté de mise en œuvre, manque d'implication des services fonciers, etc.) et dénotant une centralisation du processus décisionnel qualifiée d'excessive.

33 À cela s'ajoutent des conflits de temporalité, décentralisation des pouvoirs et gestion durable des ressources naturelles étant deux processus qui ne peuvent se conjuguer sur une échelle de temps compatible avec celle des projets ou programmes actuels des opérateurs et organismes d'aide. Des conflits de temporalité sont donc présents dans toutes les formes de coopération: qu'elles soient classiques ou décentralisées (surtout quand cette dernière dépend de sources de financement extérieurs, octroyés par les bailleurs par exemple). Belvaux (2006) souligne également le déficit d'encadrement des collectivités territoriales et des structures de regroupement local (ce qui illustre également une défaillance de la coopération décentralisée à Madagascar), l'incapacité de l'administration et de ses services déconcentrés à produire des documents de planification (zonage et aménagements forestiers), de nombreuses carences en matière de contrôle des flux (produits forestiers ligneux et non ligneux) et un laxisme dans le recouvrement de l'impôt (taxes et redevances) : «la restauration d'un 'État de droit' constitue la pierre angulaire pour améliorer le système. Une administration forte, dotée de moyens de contrôle adaptés, une gouvernance locale autonome ne subissant pas de pressions politiques exogènes, la mise en place d'une fiscalité 'de proximité' permettant au citoyen de voir comment est utilisé et à qui profite l'impôt figurent parmi les principaux axes de travail à encourager » (ibid. : 7).

34 De manière générale, la gouvernance décentralisée des ressources naturelles et de l'environnement est une problématique centrale dans les pays en développement (et ce quelle que soit la forme de coopération la soutenant). La décentralisation passe par le transfert de pouvoirs, de fonctions et de compétences de l'État à des instances locales et elle est souvent associée à l'accroissement de la démocratie locale. Elle est censée améliorer l'efficacité de la gestion des ressources naturelles grâce à la réduction de coûts de transaction et aux bénéfices perçus directement par les autorités et les populations locales. Elle est également censée favoriser l'appropriation des décisions locales par 
l'ensemble des acteurs et contribuer à la protection de l'environnement en orientant les comportements vers des pratiques plus durables.

Toutefois, plusieurs critiques émergent du constat actuel retiré de plusieurs expériences de décentralisation (Froger et Méral, 2008). Certains expliquent les écueils de la décentralisation par les dynamiques induites de " corruption décentralisée » (Véron et al., 2006), renvoyant la décentralisation face à l'un de ces principaux objectifs : rendre transparente la gestion publique, considérée comme opaque et corrompue au niveau central. Ces dynamiques de corruption décentralisée renforceraient les phénomènes de courtage en développement et les stratégies de captage de rente (Bierschenk et al., 2000).

Par ailleurs, force est de constater que la décentralisation a été souvent menée trop rapidement et de manière incomplète, laissant place à des formes hybrides plus proches de la déconcentration (Batterbury et Fernando, 2006). Enfin, Ribot et al. (2006) notent l'absence de réels transferts de pouvoirs, de mécanismes de responsabilité à l'égard de la population locale et le manque de ressources financières. Ils soulignent également que les politiques de décentralisation sont souvent "freinées" par des États, des politiciens soucieux de conserver leurs prérogatives, voire des organisations non gouvernementales de conservation dénonçant la lenteur avec laquelle les populations locales réduisent leurs pressions sur les ressources.

\section{Conclusion}

Après avoir identifié la coopération décentralisée, ses champs d'action, l'évolution de ses modalités d'intervention ainsi que les ambiguïtés auxquelles elle était confrontée en matière d'appui au développement durable dans les pays du Sud, nous avons montré que les politiques et programmes de développement durable relevaient principalement aujourd'hui des formes classiques de coopération (restant complémentaires à la coopération décentralisée sur plusieurs thématiques). Toutefois, la gouvernance décentralisée des ressources naturelles et de l'environnement promue par ces formes classiques de coopération n'en reste pas moins marquée par des ambiguïtés et est confrontée à de nombreuses difficultés, notamment à Madagascar.

La coopération décentralisée peut agir de manière complémentaire mais ses conditions de réussite dépendent, entre autres, du succès des processus de décentralisation dans les pays du Sud; par ailleurs les structures locales doivent disposer de capacités institutionnelles suffisantes pour l'élaboration et la mise en place de programmes s'inscrivant dans une perspective de plus long terme afin d'assurer démocratie et durabilité. 


\section{BIBLIOGRAPHIE}

Adriamahafazafy F., Meral Ph., 2004, « La mise en œuvre des plans nationaux d'action environnementale : un renouveau des pratiques des bailleurs de fonds? ", Mondes en développement, Vol. 32, n¹27, pp. 27-42.

Batterbury S., Fernando J., 2006, "Rescaling Governance and the Impacts of Political and Environmental Decentralization: An Introduction", World Development, Vol. 34, n¹1, pp. 1851-1863.

Belvaux E., 2006, « Décentralisation et gestion des ressources naturelles dans un pays en développement : l'exemple de Madagascar ", Communication au colloque "Gestion concertée des ressources naturelles et de l'environnement" (GECOREV), Université de Versailles-Saint-Quentin-enYvelines, 26-28 juin.

Belvaux E., Rabearisoa A., 2006, «Valorisation économique, exploitation raisonnée, utilisation durable et transfert de gestion des ressources naturelles aux communautés de base : quelle compatibilité ? ", Communication au colloque "Gestion concertée des ressources naturelles et de l'environnement" (GECOREV), Université de Versailles-Saint-Quentin-en-Yvelines, 26-28 juin. Bierschenk T., Chauveau J.-P., Olivier de Sardan J., 2000, Courtiers en développement. Les villages africains en quête de projets, Paris, Karthala.

Bonnin M., 2008, Les corridors écologiques. Vers un troisième temps du droit de la conservation de la nature?, Paris, L'Harmattan.

Chaboud C., Froger G., Méral Ph., 2009, « L'expérimentation du développement durable à Madagascar : réalités et difficultés ", Mondes en développement, Vol. 37, n¹48, pp. 47-66 (numéro coordonné par B. Boidin et A. Djeflat).

DGCID, 2006, Coopération décentralisée, tourisme responsable et solidaire et développement des territoires, Paris, MAE, $94 \mathrm{p}$.

Froger G., 2006, «Significations et ambiguïtés de la gouvernance dans le champ du développement durable », Mondes en développement, Vol. 34, n¹36, pp. 11-28.

Froger G., Adriamahafazafy F., 2003, « Les stratégies environnementales des organisations internationales dans les pays en développement : continuité ou ruptures ? », Mondes en développement, Vol. $31, \mathrm{n}^{\circ} 124$, pp. 49-76.

Froger G., Méral Ph., 2008, « Environnement et décentralisation dans les pays en développement (introduction) », Mondes en développement, Vol. 36, n¹41, pp. 7-10.

Froger G., Méral Ph., 2009, «Les temps de la politique environnementale à Madagascar : entre continuité et bifurcations ", in : G. Froger, V. Géronimi, Ph. Méral et P. Schembri (dir.), Diversité des politiques de développement durable. Temporalités et durabilités en conflit à Madagascar, au Mali et au Mexique, Paris, Karthala, pp. 45-67.

Froger G., Méral Ph., Herimandimby V., 2004, "The expansion of participatory governance in the environmental policies of developing countries: the example of Madagascar", International Journal of Sustainable Development, Vol. 7, n², pp. 164-184. 
Houzir M., 2007, « La coopération décentralisée : dynamique actuelle et perspectives », Revue Liaison Énergie-Francophonie, 77(4), pp. 16-21.

IEPF, 2007, Coopération décentralisée et développement durable, numéro spécial de la Revue Liaison Energie-Francophonie, 77(4), 101 p.

Joly A., Gallet B., 2006, « Le tourisme responsable, enjeu majeur de la coopération décentralisée. Panorama des coopérations décentralisées pour un tourisme responsable », Revue Espaces, $\mathrm{n}^{\circ} 233$, $8 \mathrm{p}$.

Marie A., 2005, La coopération décentralisée et ses paradoxes, Paris, Karthala.

Pettiteville F., 1995, La coopération décentralisée. Les collectivités locales dans la coopération Nord-Sud, Coll. Logiques politiques, Paris, L'Harmattan.

Ribot J., Agrawal A., Larson A., 2006, "Recentralizing While Decentralizing: How National Governments Reappropriate Forest Resources", World Development, Vol. 34, n¹1, pp. 1851-1863.

Tulard M.J., 2006, La coopération décentralisée, Paris, LGDJ.

Véron R., Williams G., Corbridge S., Srivastava M., 2006, "Decentralized Corruption or Corrupt Decentralization? Community Monitoring of Poverty- Alleviation Schemes in Eastern India ", World Development, Vol. 34, n¹1, pp. 1922-1941.

\section{NOTES}

1. Si la loi de décentralisation de 1982 n'a mentionné que de manière minimaliste la coopération décentralisée (en visant seulement les actions de coopération transfrontalière des régions), la loi d'administration territoriale de la République du 6 février 1992 a au contraire permis une véritable reconnaissance législative aux actions de coopération extérieure des collectivités territoriales et de leurs groupements, en systématisant le recours à une convention.

2. Au sens de coopération décentralisée au sens large.

3. Tulard (2006) insiste non seulement sur le cadre juridique encadrant la coopération décentralisée (et les ambiguïtés qu'il comporte) en France, mais également sur la diversité des acteurs en appui à cette coopération (qu'elle décrit de manière complète) : l'État, l'Europe, les associations (aux niveaux national et local), les cofinancements et les bailleurs de fonds.

4. Par exemple les jumelages entre les communes françaises et allemandes ont été une des premières formes contemporaines de coopération infra-étatique, au lendemain de la seconde guerre mondiale, pour resserrer les liens entre les deux pays.

5. Pour une analyse complète de la coopération décentralisée française, voir le site internet de la CNCD, http://cncd.diplomatie.gouv.fr/frontoffice/index.asp.

6. Source : http://cncd.diplomatie.gouv.fr/frontoffice/index.asp.

7. Le volet «développement rural » concerne principalement le développement de l'Afrique, même si l'antériorité des interventions revient aux ONG françaises (Petiteville, 1995). Les actions dans le domaine agricole concernent: l'irrigation et l'hydraulique agricole, l'amélioration des techniques culturales, la valorisation de la chaîne de production agricole... Les préoccupations environnementales et de développement durable sont également très présentes ; elles portent, entre autres, sur les mesures de protection contre diverses atteintes à l'environnement (déforestations, appauvrissement des sols, dissémination des déchets, etc.). « Les parcs naturels régionaux sont également très présents et ont développé des partenariats pour aider la délimitation de zones protégées mettant en valeur le patrimoine naturel des régions concernées et favorisant un tourisme de nature respectueux des valeurs et des cultures locales et porteur de 
développement. Ces actions sont portées par les collectivités territoriales comprises dans le territoire du parc» (Tulard, $2006: 105)$.

8. Les États et les organismes multilatéraux soutiennent des projets de coopération décentralisée dans le domaine de l'appui institutionnel en faveur du développement durable, suite à l'adoption de la notion d'Agenda 21 issu de la Conférence de Rio en 1992 et suite aux engagements pris lors du Sommet mondial du développement durable à Johannesburg en 2002.

9. Houzir (2007) note également le décalage entre les motivations des collectivités du Nord (d'ordre plutôt politique) et celles des partenaires du Sud (plus pragmatiques). En matière de coopération décentralisée, il s'agit bien d'une relation dans le sens Nord-Sud; si les échanges entre collectivités « riches » et collectivités " pauvres » ne sont pas forcément à sens unique, ils n'en restent pas moins inégalitaires.

10. Sur l'évolution des stratégies environnementales sous-jacentes aux formes classiques de coopération, voir Froger et Andriamahefazafy et Froger (2003), ainsi que Andriamahefazafy et Méral (2004).

11. Sur ce point voir l'analyse détaillée de Froger et Méral (2009).

12. À partir de la fin des années 1990, à Madagascar, la détermination collective, par les populations locales, des priorités de développement aux niveaux communal et régional (pratique qui va bien au-delà de la seule gestion décentralisée des ressources) devient également un outil de gouvernance décentralisée : les processus de négociation ne portent pas sur les modalités d'un transfert de gestion mais sur celles de l'établissement de priorités à privilégier au sein de politiques de développement en milieu rural (Froger et al., 2004). Le champ d'action est alors élargi à l'ensemble des perspectives de développement local et non plus seulement à la gestion d'une ressource donnée (comme les ressources forestières, par exemple). Voir Froger (2006) pour une analyse critique de cette forme de gouvernance décentralisée. Voir également Chaboud et al. (2009) pour une analyse critique de la planification éco-régionale, autre forme de gouvernance décentralisée promue dans la cadre de la politique environnementale malgache et soutenue par les formes classiques de coopération.

13. La GELOSE s'insère dans un cadre plus général de textes relatifs à la décentralisation, à la création des communes et des provinces autonomes: loi 93-005 du 28 janvier 1994 portant orientation générale de la politique de décentralisation; loi 94-007 du 26 avril 1995 relative aux pouvoirs, aux compétences et ressources des collectivités locales décentralisées ; loi 94-008 du 26 avril 1995 fixant les règles relatives à l'organisation, au fonctionnement et aux attributions des collectivités territoriales décentralisées.

\section{RÉSUMÉS}

Après avoir identifié la coopération décentralisée, ses champs d'action, l'évolution de ses modalités d'intervention ainsi que les ambiguïtés auxquelles elle est confrontée en matière d'appui au développement durable dans les pays du Sud, nous nous focalisons sur les politiques et programmes de développement durable relevant principalement de formes plus classiques de coopération. Toutefois, la gouvernance décentralisée des ressources naturelles et de l'environnement promue par ces formes classiques de coopération est confrontée à de nombreuses difficultés, notamment à Madagascar. La coopération décentralisée peut agir de manière complémentaire mais ses conditions de réussite dépendent, entre autres, du succès des processus de décentralisation dans les pays du Sud. 
After identifying decentralized cooperation, its fields of action, changes in its procedures and the ambiguities it faces in supporting sustainable development in developing countries, we focus on sustainable development policies and programs based on more conventional forms of cooperation. However, the decentralized governance of natural resources and environment promoted by these conventional forms of cooperation is facing many difficulties, particularly in Madagascar. Decentralized cooperation can be complementary but its conditions for success depend, among other things, on the success of decentralization processes in the South.

\section{INDEX}

Keywords : decentralization, decentralized governance, Forms of cooperation, Madagascar, natural resources, sustainable development

Mots-clés : décentralisation, développement durable, gouvernance décentralisée, Madagascar, ressources naturelles

\section{AUTEUR}

\section{GÉRALDINE FROGER}

Economiste, maître de conférences, habilitée à diriger des recherches, à l'Université de Versailles-Saint-Quentin-en-Yvelines, où elle effectue ces recherches au sein du CEMOTEV (Centre d'études sur la mondialisation, les conflits, les territoires et les vulnérabilités, EA 4457). geraldine.froger@uvsq.fr 